\title{
Coarse-grained field wave function in stochastic inflation
}

\author{
Mauricio Bellini* \\ Instituto de Física y Matemáticas, Universidad Michoacana de San Nicolás de Hidalgo, \\ AP: 2-82, (58041) Morelia, Michoacán, México
}

\begin{abstract}
The wave function for the matter field fluctuations in the infrared sector is studied within the framework of inflationary cosmology. These fluctuations are described by a coarse-grained field which takes into account only the modes with wavelength much bigger than the size of the Hubble horizon. The case of a power-law expanding universe is considered and it is found that the relevant phase-space $\left(\phi_{c g}, P_{\phi_{c g}}\right)$ remains coherent under certain circumstances. In this case the classical stochastic treatment for matter field fluctuations is not valid, however, for $p>4.6$, the system loses its coherence and a classical stochastic approximation is allowed.
\end{abstract}

PACS number(s): 98.80.Cq, 04.62.+v

Typeset using REVTEX

*E-mail address: mbellini@mdp.edu.ar 


\section{INTRODUCTION}

A standard mechanism for galaxy formation is the amplification of primordial fluctuations by the evolutionary dynamics of spacetime. The inflationary cosmology is based on the dynamics of a quantum field undergoing a phase transition [1]. The exponential expansion of the scale parameter gives a scale-invariant spectrum naturally. This is one of the many attractive features of the inflationary universe, particulary with regard to the galaxy formation problem [2] and it arises from the fluctuations of the inflaton, the quantum field which induces inflation. This field can be semiclassically expanded in terms of its expectation value plus other field, which describes the quantum fluctuations [3]. The quantum to classical transition of quantum fluctuations has been studied in thoroughly [4.

The infrared matter field fluctuations are classical and can be described by a coarsegrained field which takes into account only wavelengths larger than the Hubble radius. The dynamics of this coarse-grained field is described by a second order stochastic equation, which can be treated using the Fokker-Planck formalism. This issue has been the subject of intense work during the last two decades [5] 8]. A different approach is the quantum mechanical treatment of the coarse-grained field [9], where the fluctuations are described by means of a time dependent quadratic potential with a linear external stochastic force. Even though an isolated system described by the Schrödinger equation cannot lose its coherence, the coase-grained field may evolve from a pure to a mixed state. One way to realize coarsegraining is to let the system interact with an environment. This consists of all the fields whose evolution we are not interested in. The state of the system is obtained by tracing over all possible states of the environment. Even if the state describing the system plus environment is pure, the state of the system alone will in general be mixed. This is the case of the matter field fluctuations in inflationary cosmology, where the system is given by the super Hubble modes (infrared sector) while the environment is given by the shortwavelength modes (ultraviolet sector). For a supercooled expansion of the universe the environment cannot be considered as a true environment because it is not thermalized. A true environment appears in warm and fresh inflationary scenarios [10,11] where the inflaton field interacts with other particles of a thermal bath. In these scenarios the environment is represented by the thermal bath and the particles in it.

In this work, we aim at studying the phase-space decoherence of the wave function that describes super Hubble matter field fluctuations during inflation. Decoherence of the phasespace is different to decoherence of the coarse-grained field. While in the latter coarse-grained field decoherence consists in the interchange of degrees of freedom between the infrared and ultraviolet sectors, in the former there is interference between the fluctuations of variables that describe the phase-space of the quantum state. This issue has been treated before using either the Wigner function [12] or the Schrödinger formalism. It is well known that the evolution of the redefined coase-grained field is described by a second-order stochastic equation. This is considered in [6] for supercooled inflation and also in [13] for warm inflation. The effective Hamiltonian related to this stochastic equation can be expressed in such away that the Schrödinger equation for the system can be written. The wave function that describes this system is $\Psi\left(\chi_{c g}, t\right)$, where $\chi_{c g}$ denotes the coordinate and the variable $P_{c g} \equiv \dot{\chi}_{c g}$ characterizes the momentum of the phase-space. The interference between the the squared

fluctuations $\left\langle\chi_{c g}^{2}\right\rangle$ and $\left\langle P_{c g}^{2}\right\rangle$, which arises from the coupling between the variables $\chi_{c g}$ and 
$P_{c g}$ is the main feature to be analyzed in this paper. It has been shown that in a de Sitter expansion of the universe the phase-space of the quantum state remains pure [9]. In this work the decoherence in the phase-space for a power-law expanding universe in a globally Friedmann-Robertson-Walker (FRW) metric is studied.

The paper is organized as follows: in section II, a revision of the inflationary formalism is done. In section III, it is introduced the general quantum mechanical formalism for the coarse-grained field, which describe the redefined matter field fluctuations on super Hubble scales. In section IV, the wave function for the particular case of a power-law expansion of the universe is studied. Finally, in section V, some final remarks are given.

\section{REVIEW OF THE INFLATIONARY FORMALISM}

In a previous work [6] we justify the classical behaviour of the order parameter on the basis of a semiclassical approach. The inflaton field Lagrangian is:

$$
\mathcal{L}(\varphi, \varphi, \mu)=-\sqrt{-g}\left[\frac{1}{2}\left(g^{\mu \nu} \varphi_{, \mu} \varphi_{, \nu}\right)+V(\varphi)\right]=a^{3}\left(\frac{1}{2} \dot{\varphi}^{2}-\frac{1}{2 a^{2}}(\nabla \varphi)^{2}-V(\varphi)\right),
$$

for a globally flat FRW metric, $d s^{2}=-d t^{2}+a(t)^{2} d \vec{r}^{2}$. From here it follows the equation for the scalar field operator

$$
\ddot{\varphi}-\frac{1}{a^{2}} \nabla^{2} \varphi+3 H \dot{\varphi}+V^{\prime}(\varphi)=0,
$$

and the Friedmann equation, written in terms of $H=\frac{\dot{a}}{a}$, is

$$
H^{2}=\frac{4 \pi}{3 M_{p}^{2}}\left\langle\dot{\varphi}^{2}+\frac{1}{a^{2}}(\vec{\nabla} \varphi)^{2}+2 V(\varphi)\right\rangle,
$$

where the overdot represents the time derivative and $V^{\prime}(\varphi)=\frac{d V}{d \varphi}$. We decompose the scalar field as its mean value plus the quantum spatially inhomogeneous fluctuations, $\varphi(\vec{x}, t)=$ $\phi_{c l}(t)+\phi(\vec{x}, t)$ with $\langle\phi\rangle=0$, up to linear terms in $\phi$. Hence, the equations of motion reduce to a set of two classical equations which give the evolution of the field $\phi_{c l}$ and the Hubble parameter. For simplicity, we consider the Hubble parameter as classical: $H \equiv H\left(\phi_{c}\right)=\frac{\dot{a}}{a}$. To be consistent with the FRW metrics, it is assumed that $\phi_{c l}$ is a homogeneous field, and thus we have:

$$
\begin{aligned}
& \ddot{\phi}_{c l}+3 H \dot{\phi}_{c l}+V^{\prime}\left(\phi_{c l}\right)=0, \\
& H^{2}=\frac{8 \pi^{2}}{3 M_{p}^{2}} \rho,
\end{aligned}
$$

where $V^{\prime}\left(\phi_{c l}\right)=\frac{d V\left(\phi_{c l}\right)}{d \phi_{c l}}$ and $\rho=\frac{1}{2} \dot{\phi}_{c l}^{2}+V\left(\phi_{c l}\right)$ is the vacuum energy density. The equation for the quantum fluctuations is

$$
\ddot{\phi}-\frac{1}{a^{2}} \nabla^{2} \phi+3 H \dot{\phi}+V^{\prime \prime}\left(\phi_{c l}\right) \phi=0 .
$$

In this last equation $H\left(\phi_{c l}\right)$ and $V^{\prime \prime}\left(\phi_{c l}\right)$ are given by equations (困) and (5), and they both are functions of $t$. 
The characteristic timescale for the inflaton field can be defined by $\tau_{d}=\frac{\phi_{c l}}{\dot{\phi}_{c l}}$. The Hubble timescale is given by:

$$
\nu \equiv \frac{\tau_{d}}{\tau_{H}}=\tau_{d} H=\frac{H \phi_{c l}}{\dot{\phi}_{c l}}=\sqrt{\frac{2}{3}} \frac{2 \pi}{M_{p}} \frac{\phi_{c l}}{\dot{\phi}_{c l}} \rho^{1 / 2} .
$$

The number of e-folds in a given period of time is given by:

$$
N_{c}=\int_{t_{0}}^{t_{0}+\delta t} d t H=\int_{\phi_{0}}^{\phi_{c l}} d \phi_{c l}^{\prime} \frac{\nu}{\phi_{c l}^{\prime}}
$$

When the scalar field potential is sufficiently flat the field rolls to the minimum of the potential very slowly, and the following conditions are fulfilled

$$
\begin{aligned}
& \Theta=\frac{2}{K^{2}}\left(\frac{H^{\prime}}{H}\right)^{2} \ll 1, \\
& \Sigma=\frac{2}{K^{2}} \frac{H^{\prime \prime}}{H} \ll 1,
\end{aligned}
$$

with $K=\frac{\sqrt{8 \pi}}{M_{p}}$ [14. When the slow-roll conditions hold, the eq. (6) can be approximated by a first-order equation of motion for $\phi$ [15]. However, in this paper we will consider the exact treatment for the dynamics of $\phi$. At the end of inflation, when the scale factor stops accelerating, one obtains $\Theta\left(\phi_{c l}\right)=1$, which determines $\phi_{c l}^{e n d}$.

Hence, we have $\dot{\phi}_{c l} \simeq-\frac{V^{\prime}\left(\phi_{c l}\right)}{3 H}$ and $H^{2}=\frac{8 \pi^{2}}{3 M_{p}^{2}} V\left(\phi_{c l}\right)$, so that:

$$
\nu \sim-\frac{8 \pi^{2} \phi_{c l}}{M_{p}^{2}} \frac{V\left(\phi_{c l}\right)}{V^{\prime}\left(\phi_{c l}\right)}
$$

and

$$
N_{c}=-\frac{8 \pi^{2}}{M_{p}^{2}} \int_{\phi_{0}}^{\phi_{c l}} d \phi_{c l}^{\prime} \frac{V\left(\phi_{c l}^{\prime}\right)}{V^{\prime}\left(\phi_{c l}^{\prime}\right)} .
$$

The solution to the horizon problem requires $N_{c} \gtrsim 60$, which in general implies that $\tau_{d}>\tau_{H}$. When that scale crosses the Hubble radius, each length scale $\Omega_{c}$ is associated with a unique value of $\phi_{c l}$ denoted by $\Omega\left(\phi_{c l}\right)$. This is given by [14]:

$$
\Omega_{c}\left(\phi_{c l}\right)=\frac{\exp \left[N_{c}\left(\phi_{c l}\right)\right]}{H\left(\phi_{c l}\right)} \frac{a_{o}}{a_{e}},
$$

with $a\left(\phi_{c l}\right)=a_{e} \exp \left[-N_{c}\left(\phi_{c l}\right)\right]$.

The study of the quantum component is simplified if we redefine the field $\phi$ with the map $\phi=e^{-\frac{3}{2} \int d t H} \chi$. The equation of motion for the field operator $\chi$ is:

$$
\ddot{\chi}-\frac{1}{a^{2}} \nabla^{2} \chi-\frac{k_{0}^{2}}{a^{2}} \chi=0,
$$

where $k_{0}^{2}=a^{2}\left(\frac{9}{4} H^{2}+\frac{3}{2} \dot{H}-V_{c}^{\prime \prime}\right)$. Thus $\chi$ can be interpreted as a free scalar field with a time dependent mass parameter. The field $\chi$ can be expanded in a set of modes $\xi_{k}(t) e^{i \vec{k} \cdot \vec{r}}$ : 


$$
\chi(\vec{r}, t)=\frac{1}{(2 \pi)^{3 / 2}} \int d^{3} k\left[a_{k} \xi_{k}(t) e^{i \vec{k} \cdot \vec{r}}+\text { h.c. }\right]
$$

where the annihilation and creation operators satisfy the usual commutation relations for bosons:

$$
\begin{aligned}
& {\left[a_{k}, a_{k^{\prime}}^{\dagger}\right]=\delta^{(3)}\left(\vec{k}-\vec{k}^{\prime}\right)} \\
& {\left[a_{k}, a_{k^{\prime}}\right]=\left[a_{k}^{\dagger}, a_{k^{\prime}}^{\dagger}\right]=0}
\end{aligned}
$$

and the modes are defined for the equation of motion

$$
\ddot{\xi}_{k}+\omega_{k}^{2} \xi_{k}=0
$$

with $\omega_{k}^{2}=a^{-2}\left(k^{2}-k_{0}^{2}\right)$. The function $k_{0}^{2}(t)$ gives the threshold between an unstable infrared sector $\left(k^{2} \ll k_{0}^{2}\right)$, which includes only wavelengths longer than the Hubble radius, and a stable short wavelength sector $\left(k^{2} \gg k_{0}^{2}\right)$. We adopt the normalization condition $\xi_{k} \dot{\xi}_{k}^{*}-\xi_{k}^{*} \dot{\xi}_{k}=i$ for the modes, such that the field operators $\chi$ and $\dot{\chi}$ satisfy the canonical commutation relations.

The coarse-grained field that describes the super Hubble spectrum contains only the modes with wavenumber smaller than the Hubble's wavenumber $k_{0}$. This field can be written as a Fourier expansion in terms of the modes

$$
\chi_{c g}(\vec{r}, t)=\frac{1}{(2 \pi)^{3 / 2}} \int d^{3} k \theta\left(k-\epsilon k_{0}\right)\left[a_{k} \xi_{k}(t) e^{i \vec{k} . \vec{r}}+h . c .\right],
$$

where $\theta$ denotes the Heaviside function and $\epsilon \ll 1$ is a dimensionless constant.

The eq. (15) for $\chi_{c g}$ can be written as

$$
\ddot{\chi}_{c g}-\mu^{2}(t) \chi_{c g}+\xi_{c}(\vec{x}, t)=0,
$$

where $\xi_{c}(\vec{x}, t)=-\epsilon\left[\frac{d}{d t}\left(\dot{k}_{0} \eta\right)+2 \dot{k}_{0} \kappa\right]$, with

$$
\begin{aligned}
& \eta=\frac{1}{(2 \pi)^{\frac{3}{2}}} \int d^{3} k \delta\left(\epsilon k_{0}-k\right)\left[a_{k} e^{i \vec{k} \cdot \vec{r}} \xi_{k}(t)+h . c .\right] \\
& \kappa=\frac{1}{(2 \pi)^{\frac{3}{2}}} \int d^{3} k \delta\left(\epsilon k_{0}-k\right)\left[a_{k} e^{i \vec{k} \cdot \vec{r}} \dot{\xi}_{k}(t)+h . c .\right]
\end{aligned}
$$

These noise arises from inflow of short-wavelength modes, produced by the cosmological evolution of both, the horizon and the scale factor of the universe. Note that in eq. (20) we have neglected the term with $-\frac{1}{a^{2}} \nabla^{2} \chi_{c g}$ because in the infrared sector the following constraint is fulfilled: $k^{2} / a^{2} \ll k_{0}^{2} / a^{2}$.

\section{WAVE FUNCTION FOR THE COARSE-GRAINED FIELD}

The effective Hamiltonian related to eq. (20) is

$$
H_{e f f}\left(\chi_{c g}, t\right)=\frac{1}{2} P_{c g}^{2}-\frac{\mu^{2}}{2} \chi_{c g}^{2}+\xi_{c} \chi_{c g}
$$


where $P_{c g}=\dot{\chi}_{c g}$ and $\mu^{2}(t)=k_{0}^{2} / a^{2}$. Hence, we can write the following Schrödinger equation

$$
i \frac{\partial}{\partial t} \Psi\left(\chi_{c g}, t\right)=-\frac{1}{2} \frac{\partial^{2}}{\partial \chi_{c g}^{2}} \Psi\left(\chi_{c g}, t\right)+\left[-\frac{\mu^{2}}{2} \chi_{c g}^{2}+\xi_{c} \chi_{c g}\right] \Psi\left(\chi_{c g}, t\right),
$$

where $\Psi\left(\chi_{c g}, t\right)$ is the wave function of the system. The probability density to find the universe with the configuration $\left(\chi_{c g}, t\right)$, is

$$
P\left(\chi_{c g}, t\right)=\Psi\left(\chi_{c g}, t\right) \Psi^{*}\left(\chi_{c g}, t\right),
$$

where the asterisk denotes the complex conjugate. An elegant way to solve the eq. (24) is based on the use of explicitely time dependent invariants of motion for time dependent quadratic potentials. Such quantities appeared in accelerator theory [16] but were first analyzed by Lewis [17]. An invariant of the form

$$
I(t)=A(t) \chi_{c g}+B(t) P_{c g}+C(t)
$$

is proposed, where the time dependent coefficients $A, B, C$ are determined by the condition

$$
\frac{\partial}{\partial t} I(t)-i\left[I(t), H_{e f f}\right]=0
$$

This requires

$$
\begin{aligned}
& \dot{A}+\mu^{2}(t) B=0, \\
& A+\dot{B}=0, \\
& \dot{C}-B \xi_{c}=0 .
\end{aligned}
$$

The equations (28) and (29) can be combined to give (for $\mu^{2}=\frac{k_{0}^{2}}{a^{2}}$ )

$$
\ddot{B}-\mu^{2} B=0
$$

and, since

$$
C(t)=\int^{t} d t^{\prime} B\left(t^{\prime}\right) \xi_{c}\left(\vec{x}, t^{\prime}\right)
$$

the only equation that we need to solve is (31). From eq. (29), the invariant now can be written as

$$
I(t)=B(t) P_{c g}-\dot{B} \chi_{c g}+C(t) .
$$

In order for $I(t)$ to be an Hermitian operator, only real solutions of eq. (31) are admissible. The eigenvalue equation for this operator is

$$
I(t) \Phi_{\lambda}(t)=\lambda \Phi_{\lambda}(t),
$$

where the eigenfunction 18

$$
\Phi_{\lambda}(t)=\frac{1}{\sqrt{2 \pi B}} e^{\frac{i}{B}\left[\frac{1}{2} \dot{B} \chi_{c g}^{2}+(\lambda-C) \chi_{c g}\right]},
$$


satisfies the orthogonality condition

$$
\left\langle\Phi_{\lambda}(t) \mid \Phi_{\lambda^{\prime}}(t)\right\rangle=\delta\left(\lambda-\lambda^{\prime}\right) .
$$

Here, the eigenvalues $\lambda$ are independent of the time. Since the action of $I(t)$ does not involve time derivatives, the function $\Phi_{\lambda}(t)$ is always arbitrary up to a time dependent phase factor. Hence, we can write the eigenfuctions of $I(t)$

$$
\psi_{\lambda}(t)=e^{i \alpha_{\lambda}(t)} \Phi_{\lambda}(t) .
$$

Taking the time derivative in eq. (37), is obtained

$$
\frac{\partial I}{\partial t} \psi_{\lambda}(t)=(\lambda-I) \frac{\partial}{\partial t} \psi_{\lambda}(t)
$$

This can be written in terms of $\Phi_{\lambda}(t)$ as

$$
\left\langle\Phi_{\lambda}(t)\left|\left(H_{e f f}-i \frac{\partial}{\partial t}\right)\right| \Phi_{\lambda}(t)\right\rangle=-\frac{\partial}{\partial t} \alpha_{\lambda}(t)\left\langle\Phi_{\lambda} \mid \Phi_{\lambda}\right\rangle,
$$

where $\alpha_{\lambda}(t)$ comes from solving

$$
\alpha_{\lambda}(t)=-\int^{t} d t^{\prime} \frac{\left[\lambda-C\left(t^{\prime}\right)\right]^{2}}{2 B^{2}\left(t^{\prime}\right)}
$$

The solutions of the time dependent Schrödinger equation are

$$
\psi_{\lambda}(t)=\frac{1}{\sqrt{2 \pi B}} e^{\frac{i}{B}\left[\frac{1}{2} \dot{B} \chi_{c g}^{2}+(\lambda-C) \chi_{c g}+\beta_{\lambda}\right]},
$$

where

$$
\beta_{\lambda}(t)=B(t) \alpha_{\lambda}(t)
$$

The general solution $\Psi\left(\chi_{c g}, t\right)$ can be represented as

$$
\Psi\left(\chi_{c g}, t\right)=\int d \eta \eta_{\lambda} \psi_{\lambda}\left(\chi_{c g}, t\right)
$$

where $\eta_{\lambda}$ the coefficients are

$$
\eta_{\lambda}=\left\langle\psi_{\lambda}\left(t_{0}\right) \mid \Psi\left(t_{0}\right)\right\rangle
$$

and $t_{0}$ is the initial time. In our case $t_{0}$ is the time when inflation starts, and corresponds to 1 in Planckian unities. We will take $\beta_{\lambda}\left(t_{0}\right)=0, B\left(t_{0}\right)=1, \dot{B}\left(t_{0}\right)=0$, and $C\left(t_{0}\right)=0$. With these choice is obtained

$$
\eta_{\lambda}=\frac{1}{\sqrt{2 \pi}} \int d \chi_{c g} e^{-i \lambda \chi_{c g}} \Psi\left(\chi_{c g}, t_{0}\right)
$$

which is the Fourier transform of the initial state. In the case of an squeezed harmonic oscillator, $\Psi\left(\chi_{c g}, t_{0}\right)$ can be written as 


$$
\Psi_{s}\left(\chi_{c g}, t_{0}\right)=\left[\frac{1}{2 \pi \sigma^{2}}\right]^{1 / 4} e^{-\left[\frac{\chi_{c g}-\chi_{c g}^{(0)}}{2 \sigma}\right]^{2}+i P_{c g}^{(0)} \chi_{c g}} .
$$

Here, $\sigma$ is the width of the gaussian and $P_{c g}^{(0)}=\dot{\chi}_{c g}\left(\vec{x}, t_{0}\right)$. By setting $z=P_{c g}^{(0)}-\lambda$, the wave function becomes

$$
\begin{aligned}
\Psi\left(\chi_{c g}, t\right) & =\left[\frac{\sigma}{\sqrt{2 \pi} \pi B}\right]^{1 / 2} e^{\frac{i}{B}\left[\frac{1}{2} \dot{B} \chi_{c g}^{2}+\left(P_{c g}^{(0)}-C\right) \chi_{c g}\right]} \\
& \times \int d z e^{-\sigma^{2} z^{2}+i\left(\chi_{c g}^{(0)}-\chi_{c g} / B\right) z+i \beta_{z} / B},
\end{aligned}
$$

where $\beta_{z}$ is given by

$$
\beta_{z}=B(t)\left[-z^{2} \mathcal{R}(t)+z \mathcal{U}(t)-\mathcal{S}(t)\right]
$$

being

$$
\begin{aligned}
& \mathcal{R}(t)=\int^{t} d t^{\prime} \frac{1}{2 B^{2}\left(t^{\prime}\right)}, \\
& \mathcal{U}(t)=\int^{t} d t^{\prime} \frac{\left[P_{c g}^{(0)}-C\left(t^{\prime}\right)\right]}{B^{2}\left(t^{\prime}\right)}, \\
& \mathcal{S}(t)=\int^{t} d t^{\prime} \frac{\left[P_{c g}^{(0)}-C\left(t^{\prime}\right)\right]^{2}}{2 B^{2}\left(t^{\prime}\right)} .
\end{aligned}
$$

In our case, $\chi_{c l}=\left\langle\chi_{c g}\right\rangle$ and $P_{c l}=\left\langle\dot{\chi}_{c g}\right\rangle$ imply that

$$
\begin{aligned}
\chi_{c g}(t) & =B(t)\left[\chi^{(0)}+\mathcal{U}(t)\right], \\
P_{c l} & =\frac{1}{B}\left[\dot{B} \chi_{c l}+P_{c g}^{(0)}-C(t)\right] .
\end{aligned}
$$

Furthermore, we can define the parameter

$$
\Delta^{2}(t)=\frac{B^{2}(t)}{\sigma^{2}}\left[\sigma^{4}+\mathcal{R}^{2}(t)\right]
$$

such that the wave function can be written as

$$
\begin{aligned}
\Psi\left(\chi_{c g}, t\right) & =\frac{1}{(2 \pi)^{1 / 4} \Delta^{1 / 2}} e^{-\frac{1}{4 \Delta^{2}}\left[\chi_{c g}-\chi_{c l}\right]^{2}} e^{i \frac{\chi_{c g}^{2}}{\Delta^{2}}\left[2 \frac{\dot{B}}{B} \Delta^{2}+\frac{\mathcal{R}(t)}{\sigma^{2}}\right]} \\
& \times e^{i \frac{\chi_{c g}}{\Delta^{2}}\left[\Delta^{2}\left(P_{c l}-\frac{\dot{B}}{B} \chi_{c l}\right)-\frac{\mathcal{R}(t) \chi_{c l}}{2 \sigma^{2}}\right]} e^{i \gamma(t)},
\end{aligned}
$$

where $\gamma(t)$ is an arbitrary phase. Furthermore, it can be verified that the expectation value of the effective energy in the infrared sector is

$$
\left\langle E_{e f f}\right\rangle=E_{c l}+\frac{1}{8 \Delta^{2}}-\frac{\mu^{2}}{2} \Delta^{2}+\frac{1}{2}\left(\frac{\dot{B}}{B} \Delta+\frac{\mathcal{R}}{2 \Delta \sigma^{2}}\right)^{2}
$$


where

$$
E_{c l}=\frac{1}{2} P_{c l}^{2}-\frac{\mu^{2}}{2} \chi_{c l}^{2}+\xi_{c} \chi_{c l}
$$

The contribution of the quantum fluctuations to $\left\langle E_{\text {eff }}\right\rangle$ is represented by the second, third, and fourth terms of (58). The fourth term describes the decoherence of the system, in such away that it is zero for coherent states and positive for decoherentized states. The parameter $\mu=k_{0} / a$ depends on the cosmological model. On the other hand, the squared fluctuations $\left\langle\chi_{c g}^{2}\right\rangle$ and $\left\langle P_{c g}^{2}\right\rangle$ are

$$
\begin{aligned}
& \left\langle\chi_{c g}^{2}\right\rangle=\chi_{c l}^{2}(t)+\Delta^{2}(t), \\
& \left\langle P_{c g}^{2}\right\rangle=P_{c l}^{2}(t)+\frac{1}{4 \Delta^{2}(t)}+\left(\frac{\dot{B}}{B} \Delta(t)+\frac{\mathcal{R}}{2 \Delta \sigma^{2}}\right)^{2} .
\end{aligned}
$$

\section{WAVE FUNCTION IN A POWER-LAW EXPANSION OF THE UNIVERSE}

If mass parameter $\mu$ is time independent but the external classical force is nonzero, the solutions are coherent states [19]. This case corresponds to a de Sitter expansion of the universe, which was studied in a previous paper by Habib and Mijić [9]. In this work we are interested in the study of the particular case of a power-law expansion of the universe in which the scale factor is $a \propto t^{p}$. In this case the squared parameter of mass is given by [6]

$$
\mu^{2}(t)=M^{2} t^{-2}
$$

where $M^{2}=\frac{9}{4} p^{2}-\frac{15}{2} p+2$ and the Hubble parameter being given by $H(t)=p / t$ [6]. The condition to get an unstable sector is $M^{2}>0$, or $p>(5+\sqrt{17}) / 3 \simeq 3.04$. Furthermore, since $H\left(\phi_{c}\right)=H_{0} e^{\phi / M_{p}}$, the slow-roll paramters $\Theta$ and $\Sigma$ become both of the order of $10^{-1}$, so that the slow-roll regime is guaranted. Hence, the equations which characterize the system are

$$
\begin{aligned}
& \ddot{B}-M^{2} t^{-2} B=0, \\
& C(t)=\int^{t} d t^{\prime} B\left(t^{\prime}\right) \xi_{c}\left(\vec{x}, t^{\prime}\right) .
\end{aligned}
$$

The general solution of eq. (63) is

$$
B(t)=c_{1} t^{\frac{1}{2}\left[1+\sqrt{1+4 M^{2}}\right]}+c_{2} t^{\frac{1}{2}\left[1-\sqrt{1+4 M^{2}}\right]},
$$

where the initial conditions $B\left(t_{0}\right)=1$ and $\dot{B}\left(t_{0}\right)=0$ imply

$$
c_{1}=\frac{\sqrt{1+4 M^{2}}-1}{2 \sqrt{1+4 M^{2}}}, \quad c_{2}=\frac{\sqrt{1+4 M^{2}}+1}{2 \sqrt{1+4 M^{2}}} .
$$

Since it is difficult to know exactly the functions $\mathcal{R}(t), \mathcal{U}(t)$ and $\mathcal{S}(t)$, we can make the calculation for late times. For $t \gg 1$, one obtains $\left.B(t)\right|_{t \gg 1} \simeq t^{1 / 2} / 2$ and 


$$
\begin{aligned}
& \left.\mathcal{R}(t)\right|_{t \gg 1} \simeq \frac{\sqrt{1+4 M^{2}}}{\left(\sqrt{1+4 M^{2}}-1\right)\left(1+\sqrt{1+4 M^{2}}\right)} \ln \left[\frac{2 \sqrt{1+4 M^{2}}}{\sqrt{1+4 M^{2}}-1}\right] \\
& \left.\Delta^{2}(t)\right|_{t \gg 1} \propto t^{1+\sqrt{1+4 M^{2}}} .
\end{aligned}
$$

This means that the decoherence function $\mathcal{D}(t)=\frac{1}{2}\left(\frac{\dot{B}}{B} \Delta+\frac{\mathcal{R}}{2 \Delta \sigma^{2}}\right)^{2}$ in the expectation effective energy (58), will be

$$
\left.\mathcal{D}(t)\right|_{t \gg 1} \propto t^{\frac{1}{2}\left(\sqrt{1+4 M^{2}}-1\right)}
$$

which always increases because $M^{2}>0$ during inflation. Replacing (67), (68) and (69) in eqs. (60) and (61) one observes that, since $\left.\Delta^{2}(t)\right|_{t \gg 1}$ increases with time, $\left\langle\chi_{c g}^{2}\right\rangle$ increases but the second term in (61) decreases as $t$ increases. The second terms in (60) and (61) describe the evolution of super Hubble fluctuations due to the exchange of degrees of freedom between the infrared $\left(k^{2} \ll k_{0}^{2}\right)$ and the ultraviolet $\left(k^{2} \gg k_{0}^{2}\right)$ sectors. The third term in eq. (61) is the most interesting one. This term describes the decoherence of the phase-space $\left(\chi_{c g}, P_{c g}\right)$ during inflation. Note [see eq. (69)], that it increases with $t$, so the phase-space loses its coherence at the end of inflation. This term comes from the coupling between $\chi_{c g}$ and $P_{c g}$. This is the physical origin of decoherence in the phase-space $\left(\chi_{c g}, P_{c g}\right)$. However, the relevant phase-space during inflation is $\left(\phi_{c g}, P_{\phi_{c g}}\right)$, where $\phi_{c g}=a^{-3 / 2} \chi_{c g}$ and $P_{\phi_{c g}}=\dot{\phi}_{c g}$. This implies that the function that describes decoherence between $\phi_{c g}$ and $P_{\phi_{c g}}$ can be written, to a good approximation, as

$$
\left.\left.\mathcal{D}\left(\phi_{c g}, P_{\phi_{c g}}, t\right)\right|_{t \gg 1} \sim a^{-3} \mathcal{D}(t)\right|_{t \gg 1} \propto t^{\frac{1}{2}\left[\sqrt{1+4 M^{2}}-(6 p+1)\right]}
$$

where $\left.\mathcal{D}(t)\right|_{t \gg 1}$ is given by (69). A numerical calculation shows that $\mathcal{D}\left(\phi_{c g}, P_{\phi_{c g}}, t\right)$ grows for $p>4.6$, but decreases in the range $3.04<p<4.6$. It is well known that power-law inflation takes place if $p>3.04$. This means that for $3.04<p<4.6$ the phase-space $\left(\phi_{c g}, P_{\phi_{c g}}\right)$ remains coherent during inflation.

\section{FINAL REMARKS}

In this paper we have considered the wave function for the coarse-grained field. It describes the redefined matter field fluctuations in the infrared sector for a globally flat FRW background metric. The Hamiltonian of the Schrödinger equation is given by an effective Hamiltonian. It has a quadratic contribution with a time dependent mass plus an effective stochastic force, which describes the "interaction" between both, ultraviolet and infrared sectors. However, this is not a true interaction in the sense of a thermalyzed environment. Here, continuosly we have new modes are crossing the ultraviolet sector increasing the number of degrees of freedom of the infrared sector. This effect appears in the stochastic equations as an effective noise $\xi_{c}$, which is responsible for the uncertaintly of the quantum state.

On the other hand the lost of coherence in the phase-space is a consequence of a nonzero correlation between the variables of such a space (in this case $\chi_{c g}$ and $P_{c g}$ ). As was shown in this work, the decoherence function $\mathcal{D}(t)$ increases with time in such away that the 
phase-space $\left(\chi_{c g}, P_{c g}\right)$ is decohered at the end of power-law inflation. However, the relevant phase-space to describe decoherence during inflation is $\left(\phi_{c g}, P_{\phi_{c g}}\right)$. We found that in the range $3.04<p<4.6$ the system does not decohere. This implies that, under these conditions, the classical treatment of super Hubble matter field fluctuations developed in stochastic inflation should be revised. On the other hand for very large $p$ (more exactly for $p>4.6)$ the phase-space $\left(\phi_{c g}, P_{\phi_{c g}}\right)$ loses its coherence at the end of inflation, and then stochastic inflation provides a good treatment for large-scale matter field fluctuations when the scale factor grows very rapidly. This calculation was performed in the framework of supercooled inflation, which does not take into account dissipative effects produced by the interaction between the inflaton field and other particles of a thermal bath. In the warm inflation [20,21], the phase-space could not either remain coherent, but as a consequence of additional thermal and dissipative effects. A more detailed treatment has to deal with complicated nonlinear effects of super Hubble fluctuations. This goes beyond the scope of this paper. I hope to consider this topic elsewhere.

\section{ACKNOWLEDGMENTS}

I would like to acnowledge F. Astorga for his careful reading of the manuscript. Also, I would like to acknowledge CONACYT (México) and CIC of Universidad Michoacana for financial support in the form of a research grant. 


\section{REFERENCES}

[1] A. H. Guth, Phys. Rev. D23, 347 (1981).

[2] for reviews on inflation see A. D. Linde, Particle Physics and Inflationary Cosmology, (Harwood Academic Publishers, New York, 1990); and references therein.

[3] A. A. Starobinsky, in Fundamental Interactions (MGPI Press, Mowcow, 1983); in Current Topics in Field Theory Quantum Gravity, and Strings edited by H. J. de Vega and N. Sánchez (Springer, New York, 1986).

[4] D. Polarski and A. A. Starobinsky, Class. Quant. Grav. 13, 377 (1996); C. Kiefer, J. Lesgourdes, D. Polarski, and A. A. Starobinsky, Class. Quant. Grav. 15, L67 (1998).

[5] S.Habib, Phys. Rev. D46, 2408 (1992).

[6] M. Bellini, H. Casini, R. Montemayor, P. Sisterna, Phys. Rev. D54, 7172 (1996).

[7] M.Mijic, Phys. Rev. D49, 6434 (1994).

[8] H. Casini, R. Montemayor and P. Sisterna, Phys. Rev. D60, 063512 (1999).

[9] S. Habib and Milan Mijić, Stochastic Dynamics of coarse-grained quantum fields in the inflationary universe, (unpublished).

[10] A. Berera, Phys. Rev. D54, 2519 (1996).

[11] M. Bellini, Fresh inflation: a warm inflationay model from a zero temperature initial state, to appear in Phys. Rev. D. E-print: gr-qc/0101062.

[12] D. Polarski, A. A. Starobinsky, Class. Quant. Grav. 13, 377 (1996); C. Kiefer, J. Lesgourgues, D. Polarski, A. A. Starobinsky, Class. Quant. Grav. 15, L67 (1997).

[13] Mauricio Bellini, Class. Quant. Grav. 16, 2393 (1999); ibid. Nucl. Phys. B563, 245 (1999).

[14] E.J.Copeland, E.W.Colb, A.R.Liddle and J.E.Lidsay, Phys. Rev. D48, 2529 (1993).

[15] see, for example, A. Mezhlumian, A. A. Starobinsky, preprint astro-ph/9406045; SooJong Rey, Nucl. Phys. B284, 706 (1987).

[16] E. D. Courant and H. S. Snyder, Ann. Phys. 3, 1(1958).

[17] H. R. Lewis, Jr., Phys. Rev. Lett. 18, 510 (1967); J. Math. Phys. 9, 1976 (1968).

[18] R. Vaidyanathan, J. Math. Phys. 23, 1346(1982).

[19] P. Carruthers and M. M. Nieto, Am. J. Phys. 33, 537(1965).

[20] A. Berera, Phys. Rev. Lett. 75, 3218 (1995).

[21] M. Bellini, Phys. Phys. Lett. B428, 31 (1998). 